\title{
Escaleras en los «nuevos» barrios populares de Lima: modalidades y entes gestores de su construcción. El caso de Horacio Zeballos ${ }^{+}$
}

\author{
KATHERIN TIBURCIO JAIMES* \\ Universidad Nacional de Ingeniería \\ katherintiburcioj@gmail.com
}

https://doi.org/108800/rcpg.201801.004

\begin{abstract}
RESUMEN
En Lima, la construcción de escaleras ha sido el caballito de batalla de más de una gestión municipal en lo que concierne a políticas urbanas en los barrios populares. Incluso, en algunas ocasiones, ha sido la única política adoptada para atender a los ciudadanos que habitan en estas áreas. Con todo ello, la dotación de escaleras se ha vuelto sinónimo de consolidación infraestructural y mejor calidad de vida en el imaginario de la mayoría de los limeños. El presente artículo busca describir los mecanismos estatales utilizados para la construcción de escaleras, abriendo el debate sobre su pertinencia y eficacia como políticas públicas, y colaborando en la elaboración de un marco más concreto sobre la temática de las políticas urbanas aplicables para los «nuevos» barrios populares de Lima.
\end{abstract}

Palabras clave: escaleras, barrios populares, espacio público, política urbana, proyectos de inversión pública.

+ Recibido el 16 de abril de 2018; aceptado el 04 de octubre de 2018.

* Bachiller en Arquitectura de la Facultad de Arquitectura, Urbanismo y Artes de la Universidad Nacional de Ingeniería. Especialista en Proyectos de Inversión Pública por la Universidad del Pacífico y en Gestión de la Movilidad Sostenible por la Fundación Mobilitat Sostenivel y Segura. 


\title{
Staircases in the «new» popular neighborhoods of Lima: modalities and management entities of its construction. The case of Horacio Zeballos
}

\begin{abstract}
In Lima, the construction of staircases has been the showpiece of many municipal governments in the field of urban policies in popular neighborhoods. On some occasions, it has been the only policy adopted to respond to citizens' demands in these areas. With all this, the provision of stairs has become synonymous with infrastructural consolidation and an improved quality of life in the imaginary of the majority of Lima residents. This article seeks to describe state mechanisms in the process of constructing public staircases, opening the debate on their relevance and effectiveness as public policies and collaborating in the elaboration of an analytical framework on urban policies applicable to the 'new' popular neighborhoods of Lima.

Keywords: staircases, popular neighborhoods, popular settlements, public space, urban policy, public investment projects.
\end{abstract}




\section{INTRODUCCIÓN}

El presente artículo es producto parcial de una investigación más amplia sobre la producción de escaleras en los nuevos barrios populares de Lima — culminada en 2015- que tomó como caso específico el estudio del sector urbano denominado Horacio Zeballos, en el distrito de Ate.

Durante la elaboración de la investigación se hallaron datos importantes sobre el contexto de las políticas públicas en las cuales se enmarcó la construcción de escaleras en Lima y que no habían sido documentados hasta el momento. Lamentablemente, estos datos no se han podido actualizar al año actual debido a que la fuente primaria ya no es de acceso público ${ }^{1}$.

Este artículo se produce con el objeto de colaborar en la elaboración de un marco más concreto sobre la temática de las políticas urbanas para los barrios populares e intenta describir los mecanismos estatales utilizados para la construcción de escaleras, abriendo el debate sobre su pertinencia y eficacia como políticas públicas.

\section{LIMA Y LOS «NUEVOS BARRIOS POPULARES»}

A partir de mediados del siglo XX, Lima ha tenido como protagonista de su urbanización a los barrios populares. Ante la ausencia de soluciones por parte del Estado sobre la creciente demanda de suelo urbano y vivienda, los ciudadanos de más bajos recursos encontraron en la producción popular de barrios una alternativa para autosatisfacer sus necesidades.

Como la «aparición» de barrios populares en Lima — desde las primeras invasiones hasta las del día de hoy - siempre ha estado condicionada a diversos factores sociales, económicos, políticos y culturales, Barreda y Ramírez Corzo (2004) identificaron que a lo largo de la historia de la expansión de la ciudad popular se han producido tres momentos a los que les corresponden respectivamente tres tipologías de barrios:

i. En un primer momento —entre 1940 y 1954 - los barrios populares se asentaron en áreas consideradas marginales en las zonas contiguas a la ciudad tradicional, dependiendo completamente de esta.

\footnotetext{
1 Con el cambio de sistema del SNIP (Sistema Nacional de Inversión Pública) al Invierte (Sistema Nacional de Programación Multianual y Gestión de Inversiones), el Banco de Proyectos ha limitado el acceso y descarga de información para ser trabajada como base de datos, al menos durante 2018.
} 
ii. En un segundo momento —entre 1954 y finales de la década de 1980, durante el boom de la expansión urbana de Lima y la exponencial necesidad de suelo y vivienda-, barrios de gran escala se asentaron en áreas alejadas de la ciudad tradicional, con la intención de conformar nuevos núcleos de expansión, dejando de lado su dependencia.

iii. En un tercer momento — desde finales de la década de 1980 hasta la actualidad, en plena escasez de suelo urbano-, los barrios populares se han vuelto a asentar en áreas consideradas marginales, desarrollando esta vez una dependencia hacia la ciudad popular más antigua y más consolidada, proveniente del segundo momento de expansión. Las áreas marginales ocupadas hoy en día no son otras que las laderas de los cerros que flanquean Lima hacia el este.

Es precisamente en el tercer momento de expansión urbana cuando aparece la denominación de «nuevos barrios populares», acuñada por Barreda y Ramírez Corzo, cuyo título de «nuevos» es bastante relativo debido a que se acuńó tanto para referirse a los barrios que empezaron a consolidarse a partir de la década de 1990 — y que actualmente tienen alrededor de treinta años de constituidos-, como a los barrios cuya producción persiste hasta el día de hoy, aunque ahora con algunos nuevos matices otorgados por el tráfico de tierras.

Los «nuevos» barrios populares, en el afán de la posesión del suelo, han ido ocupando las laderas de forma cada vez más extensiva y riesgosa, habitando zonas cuyas pendientes escarpadas no garantizan la seguridad ni de sus habitantes ni de sus viviendas.

Tan extensiva ha sido la ocupación de laderas que se calcula, a partir de datos trabajados por Barreda (2014b), que entre el 20\% y 25\% del territorio urbanizado de Lima se encuentra emplazado en laderas. Esto quiere decir que alrededor de un millón de personas ${ }^{2}$ habita actualmente en terrenos con pendientes mayores al $25 \%{ }^{3}$, siendo la mayor parte de esta población la que ocupa los «nuevos» barrios populares en condiciones de precariedad urbana y riesgo ${ }^{4}$.

\footnotetext{
2 Según cálculo propio, sobre la base de datos de Barreda (2014b) y datos sobre densidad urbana en el territorio limeño.

3 Parámetro establecido por Barreda $(2012,2014 a)$ como umbral de riesgo a partir del cual se consideran las pendientes como muy altas y con difícil habitabilidad.

4 Hay que considerar que, aunque en menor número, también hay barrios en laderas donde habitan grupos poblacionales pertenecientes a los estratos socioeconómicos más altos — como ciertos barrios de La Molina y Surco- - y que no presentan los niveles de precariedad urbana y riesgo característicos de los «nuevos» barrios populares.
} 


\section{LA CONSTRUCCión DE ESCALERAS: DE LA AUTOCONSTRUCCión A LOS PROYECTOS DE INVERSIÓN PÚBLICA}

La construcción de escaleras en los nuevos barrios populares es hoy en día una práctica aceptada - e incluso masivamente demandada - en el rubro del urbanismo en laderas. Mas, al igual que los barrios donde son construidas, su aparición no ha sido homogénea ni reciente.

Los dos agentes involucrados en la producción de escaleras actúan a través de sus propias modalidades: la población a través de la autoconstrucción y el Estado a través de la inversión pública. Sin embargo, a pesar de que la autoconstrucción está comúnmente relacionada a la falta de técnicas adecuadas de construcción y que la inversión pública está relacionada teóricamente a una oferta adecuada de capacidades técnicas, las intervenciones en torno a la construcción de escaleras en Lima han presentado diferentes matices, entre lo técnicamente apropiado y lo improvisadamente peligroso.

Figura 1. Escaleras: población y autoconstrucción $v s$. Estado e inversión pública

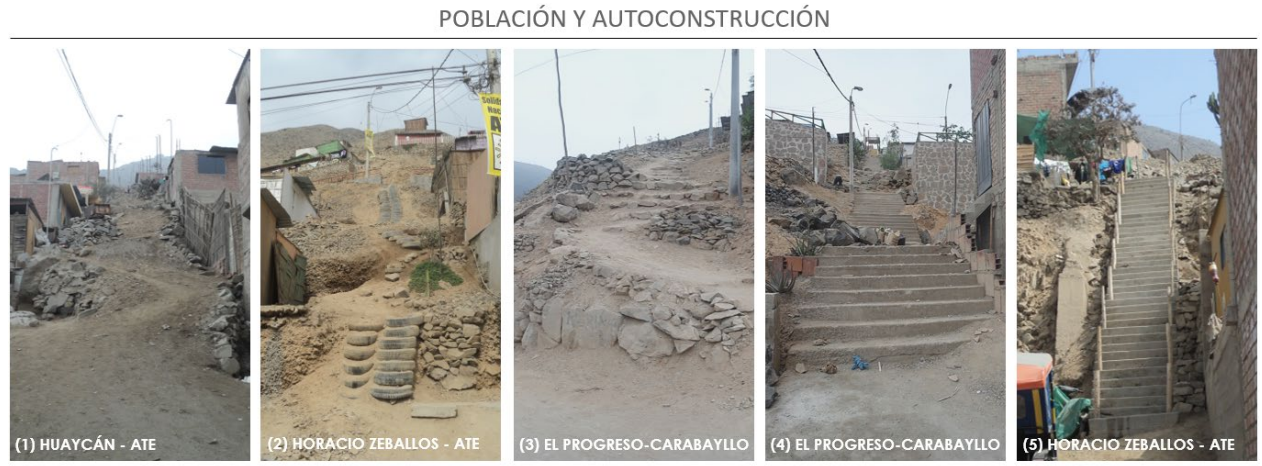

ESTADO E INVERSIÓN PÚBLICA
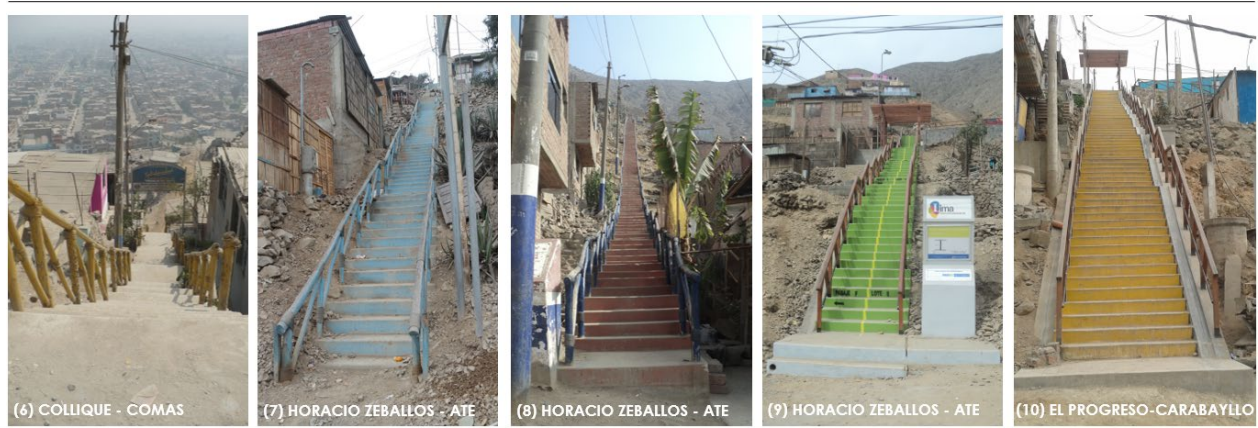

Fuente: (1) archivo propio, 2014; $(2,5,7,8,9)$ archivo propio, 2015; $(3,4,10)$ archivo propio, 2016; (6) archivo BarrioMio, 2012. Elaboración propia. 
La población, como principal agente productor de los nuevos barrios populares, ha intervenido en el espacio de la calle inmediatamente después de tener certeza del éxito de la ocupación del suelo, motivada — u obligada - por el gran problema de accesibilidad propio de los terrenos en pendiente como los que se han tenido que ocupar. Esto quiere decir que la construcción de escaleras en los nuevos barrios populares - $\mathrm{o}$ de algunos formatos incipientes parecidos a ellas - marcó el inicio del proceso de consolidación del espacio barrial.

Por parte del Estado no se tiene certeza de cuándo inició realmente la práctica de construir escaleras. Según algunos relatos informales de dirigentes y vecinos, inicialmente hubo gestiones municipales que colaboraron extraoficialmente con estas construcciones debido a alianzas o promesas pactadas previamente con la población. Fue recién a partir de la implementación del Sistema Nacional de Inversión Pública (SNIP), en el año 2000, que se comenzó a tener registro claro de las intervenciones del Estado en general y de la construcción de escaleras en particular.

Según estos registros (sistematizados y estandarizados para la elaboración de la investigación), las primeras intervenciones del Estado mediante proyectos con metas explícitas de "construcción de escaleras» aparecieron en 2004. El primero de estos proyectos se registró a finales de aquel año y estuvo promovido por la Municipalidad Distrital de Independencia. Contemplaba la construcción de una escalera en el Asentamiento Humano El Conquistador, además de la construcción de muros de contención en vías aledañas.

A partir de 2004, el número de proyectos relacionados a la construcción de escaleras $^{5}$ registrados en el Banco de Proyectos del SNIP ha ido en aumento. Hasta finales de 2015 la cantidad ascendía a 531 proyectos $^{6}$, encontrando su mayor pico en el año 2008 con alrededor de 123 proyectos al año (Figura 2). Es necesario tener en cuenta que un solo proyecto puede contemplar la construcción de más de una escalera, por lo que la existencia de 531 proyectos no significa necesariamente que solo se haya construido -o se haya planteado construir - 531 escaleras durante este tiempo, pudiendo duplicarse o hasta triplicarse este número, dependiendo de las metas formuladas en cada proyecto de inversión.

\footnotetext{
Los proyectos relacionados a la construcción de escaleras contemplan dos tipos: los elaborados exclusivamente para la construcción de escaleras y los que agrupan la construcción de escaleras y muros de contención.

6 Se han considerado todos los proyectos relacionados a la construcción de escaleras que fueron registrados en el Banco de Proyectos, tengan o no expediente técnico, debido a que su concreción solo sería cuestión de tiempo y/o recursos.
} 
Figura 2. Proyectos de inversión pública para la construcción de escaleras en las calles de Lima, según ańo de registro en el Banco de Proyectos del SNIP

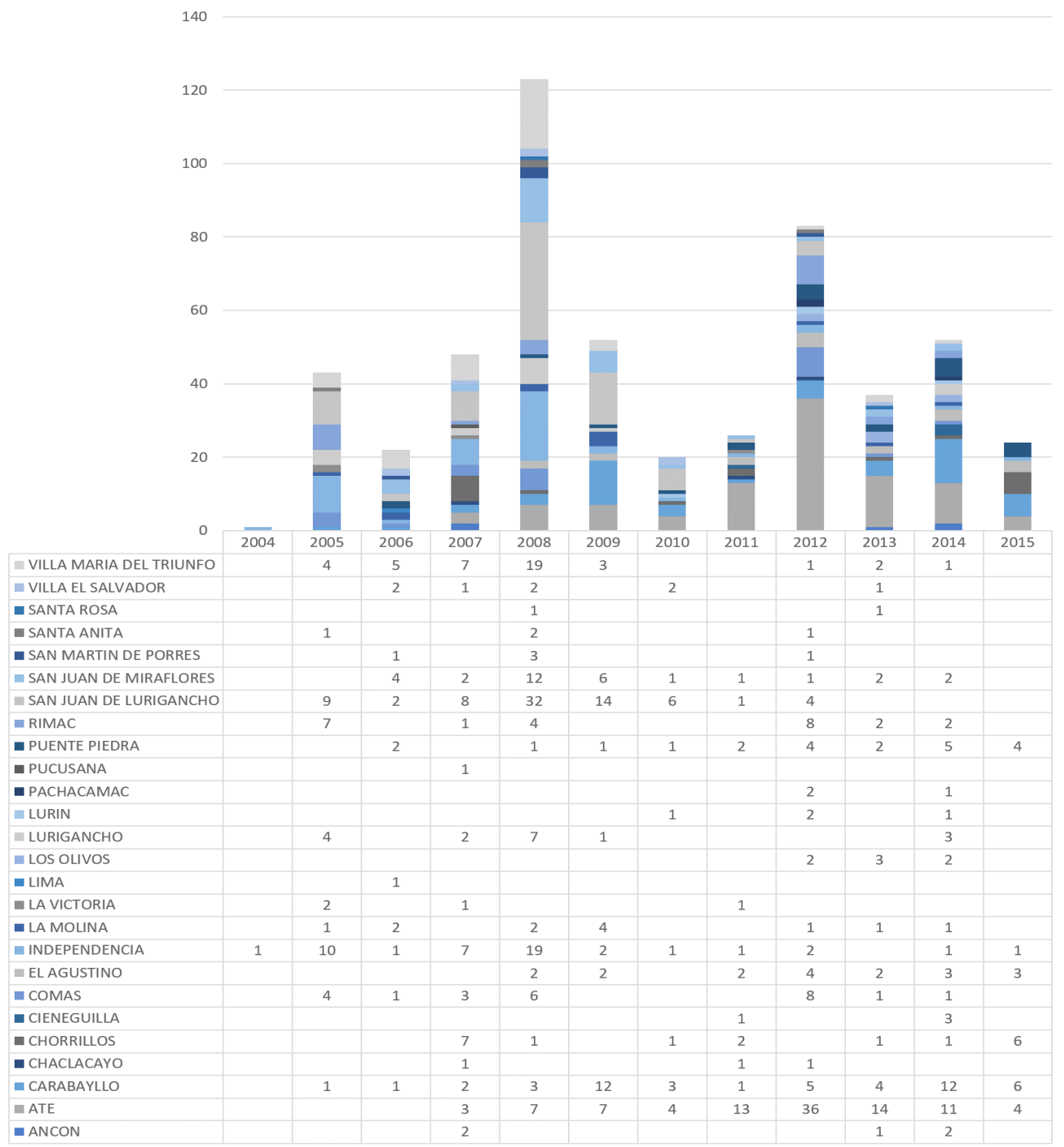

Fuente: Banco de Proyectos SNIP (fecha de corte a 2015). Elaboración propia. 
Durante el periodo 2004-2015, han sido los Gobiernos locales (regionales y distritales) los principales impulsores de este tipo de proyectos. Las intervenciones de las municipalidades distritales dieron inicio en el año 2004 y han alcanzado 263 proyectos en total (Figura 3). Mientras que las intervenciones de la Municipalidad Metropolitana de Lima — Gobierno regional—comenzaron en el año 2005, alcanzando un total de 266 proyectos (Figura 4).

El Gobierno nacional - a través del sector Vivienda, Construcción y Saneamiento- solo ha tenido actuación en la construcción de escaleras a través de dos proyectos: uno en el Rímac, registrado en 2005, y el otro en San Juan de Lurigancho, en 2007.

El cálculo de la inversión correspondiente a este tipo de proyectos se torna complejo debido a que muchos de ellos no solo contemplan la construcción de escaleras, sino que la agrupan con la construcción de muros de contención, veredas, sardineles, pistas y otros, por lo que el monto de inversión destinado exclusivamente a la construcción de escaleras es incierto y varía según las metas a alcanzar. Sin embargo, estos montos y lo que representan en la formulación de proyectos para la metodología del SNIP podrían establecer cierto panorama comparativo entre las estrategias de formulación de los Gobiernos locales y el Gobierno regional de Lima.

Teniendo en cuenta los montos determinados como umbrales por el Ministerio de Economía y Finanzas, según los cuales se requiere niveles mínimos de estudios y consideraciones para la formulación de ciertos proyectos de inversión, se propone la siguiente agrupación: Grupo 1, PIP menores con montos de inversión menores a S/ 300 000,00; Grupo 2, PIP menores con montos de inversión entre S/ 300 000,00 y S/ 1200 000,00; Grupo 3, PIP con montos mayores a S/ 1200 000,00 pero menores a S/ 20000 000,007.

A nivel de Gobiernos locales, de un total de 263 proyectos, los formulados dentro del Grupo 1 representan el 71\% (187 proyectos); del Grupo 2, el 24\% (63 proyectos); y del Grupo 3, el 5\% (13 proyectos). A nivel de Gobierno regional, de un total de 266 proyectos, los formulados dentro del Grupo 1 representan el $50 \%$ (133 proyectos); del Grupo 2, el 35\% (93 proyectos); y del Grupo 3, el 15\% ( 40 proyectos). Si bien estos datos muestran una clara tendencia de ambos niveles de gobierno por la elaboración de proyectos de poco alcance (de montos de inversión menores a S/ 300 000,00), también muestran que es el Gobierno regional de Lima el que ha apostado más por proyectos de mayor alcance y complejidad.

\footnotetext{
Estos rangos se basan en los montos determinados por el Ministerio de Economía y Finanzas, según los cuales se requiere niveles mínimos de estudios y consideraciones para su formulación.
} 
Figura 3. Proyectos de inversión pública para la construcción de escaleras en las calles de Lima a nivel de Gobiernos locales, según año de registro en el Banco de Proyectos del SNIP

60

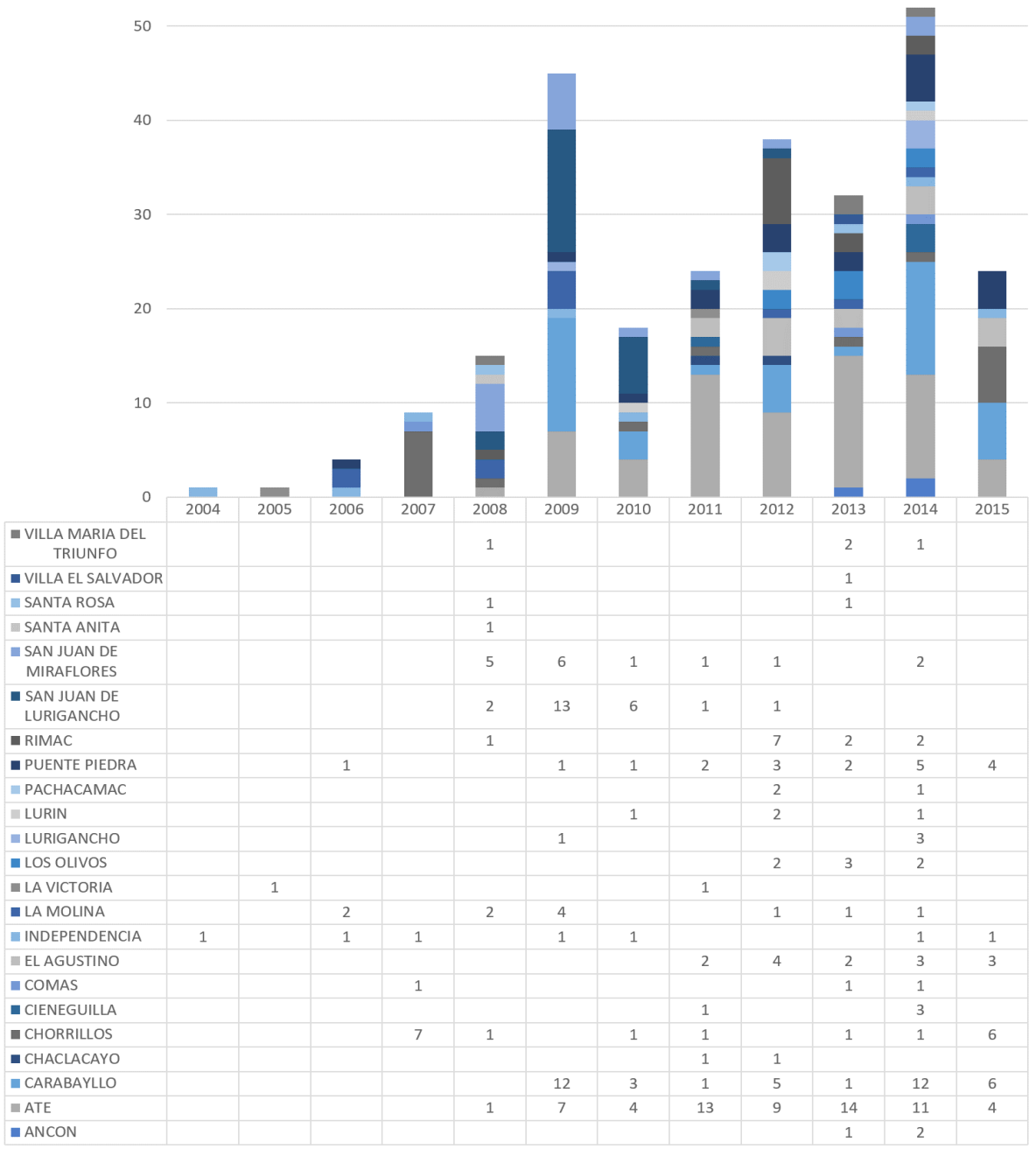

Fuente: Banco de Proyectos SNIP (fecha de corte a 2015). Elaboración propia. 
Figura 4. Proyectos de inversión pública para la construcción de escaleras en las calles de Lima a nivel de Gobierno regional, según año de registro en el Banco de Proyectos del SNIP

120

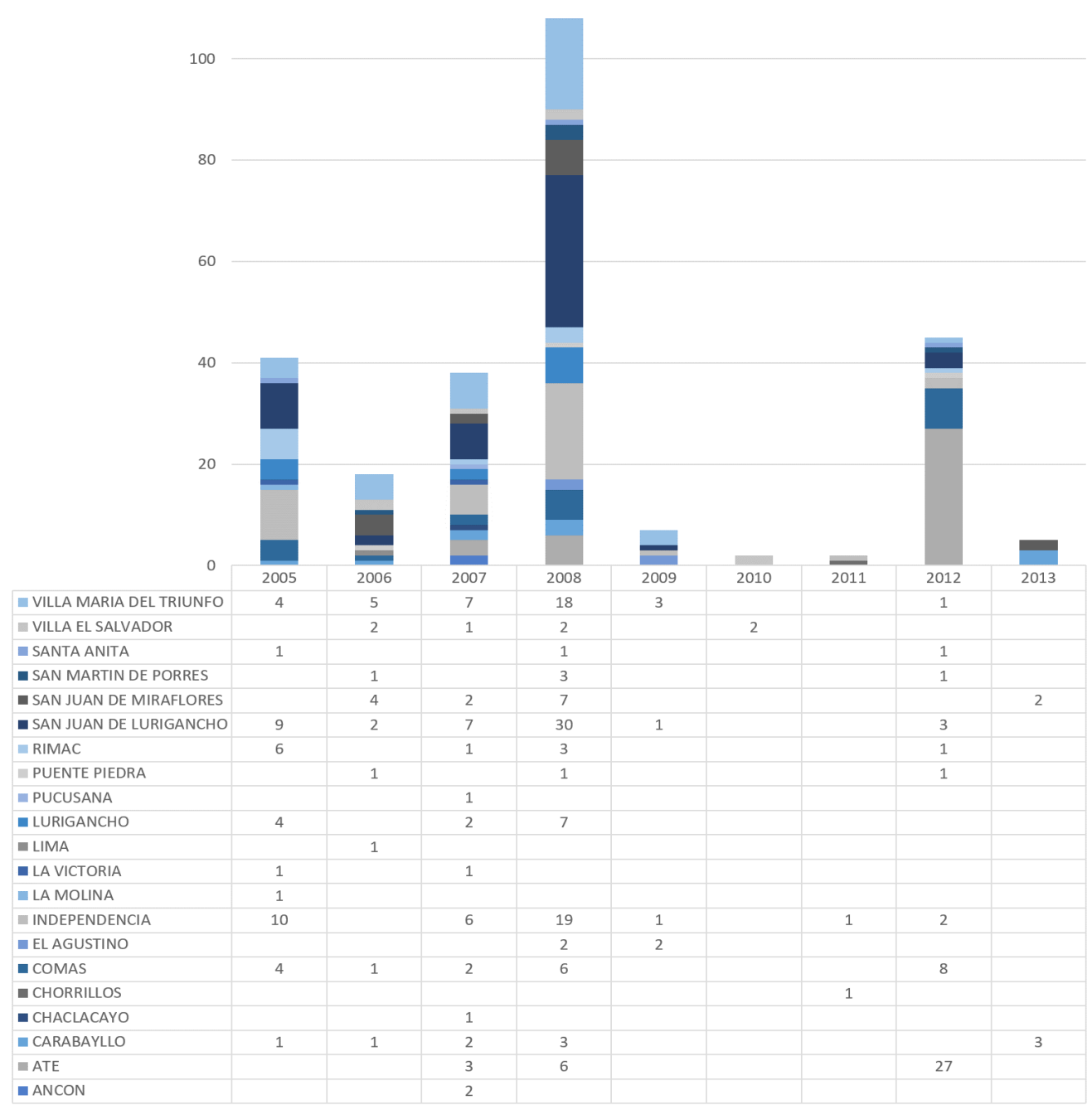

Fuente: Banco de Proyectos SNIP (fecha de corte a 2015). Elaboración propia. 
Por otro lado, con la data recopilada y sistematizada también se ha podido identificar los distritos que han sido beneficiados con la mayor cantidad de proyectos de inversión pública formulados para la construcción de escaleras (Figura 5), siendo el distrito de Ate el que ha presentado mayor cantidad de proyectos de este rubro (99), seguido de San Juan de Lurigancho (76), Carabayllo (50), Independencia (46) y Villa María del Triunfo (42).

Figura 5. Proyectos de inversión pública para la construcción de escaleras en las calles de Lima, según distritos beneficiados

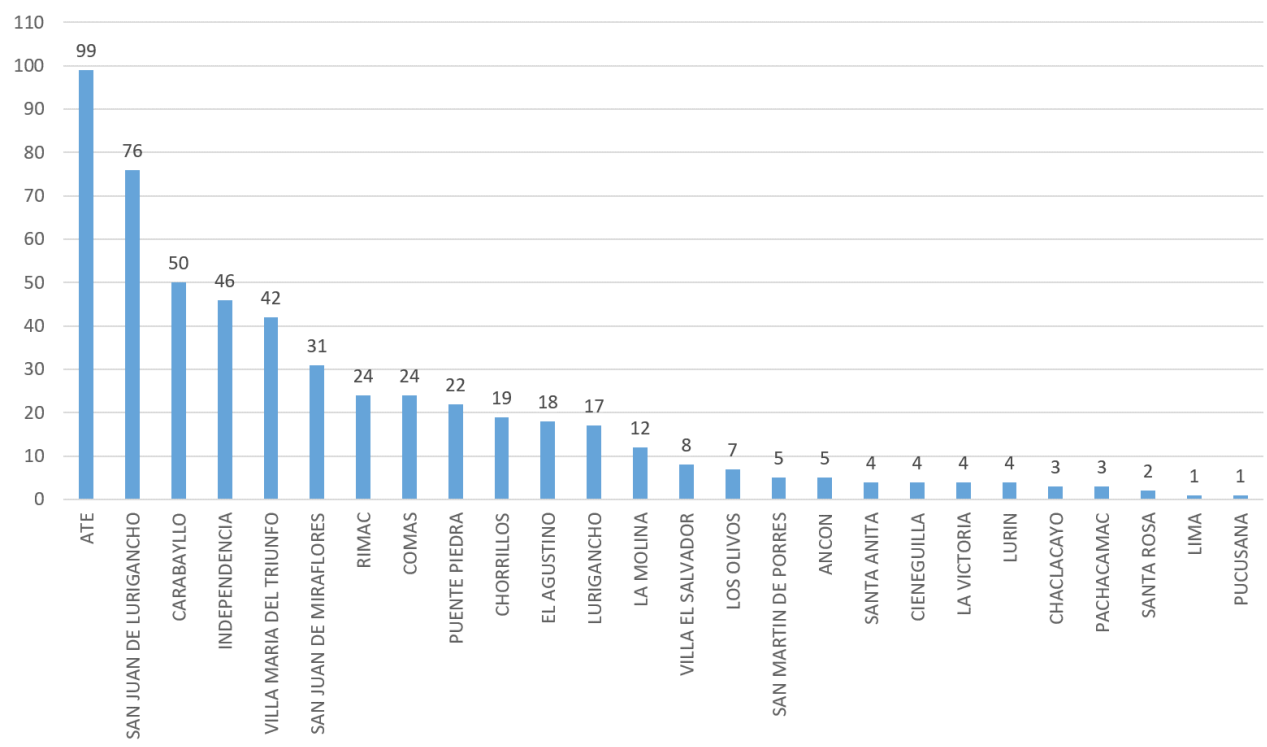

Fuente: Banco de Proyectos SNIP (fecha de corte a 2015). Elaboración propia.

\section{ESCALERAS Y GESTIONES MUNICIPALES}

La construcción de escaleras, como política urbana, también ha estado vinculada al enfoque - y al discurso - que las gestiones ediles de turno han adoptado sobre el tema. Una muestra clara y global de ello se puede apreciar en el caso de la gestión metropolitana de Lima, que durante el periodo estudiado (2004-2015) ha contado con dos estilos de gobierno municipal: el de Luis Castañeda Lossio, durante sus dos primeras gestiones (2003-2006, 2007-2010), y el de Susana Villarán de la Puente, durante su única gestión (2011-2014).

Ambas administraciones municipales desarrollaron y promocionaron públicamente programas dedicados a la construcción de escaleras — «Escaleras Solidarias» y "BarrioMio», respectivamente-, convirtiéndolos en bastiones 
discursivos de sus respectivas gestiones. Mientras el discurso de Castañeda hacía énfasis en la mejora de la accesibilidad a las viviendas, el discurso de Villarán radicaba en la mitigación del riesgo y el mejoramiento urbanístico de los barrios.

La materialización de ambos discursos en proyectos de inversión puede observarse en la elección de la función del proyecto $^{8}$ y en el tipo de alternativas de solución seleccionadas: mientras que en la gestión de Castañeda todos los proyectos se enmarcaron en la función «Transporte» y se limitaban a la dotación de infraestructura, en la gestión de Villarán la mayoría de proyectos se enmarcó en la función «Vivienda y desarrollo urbano» y sumaron al componente infraestructural la mejora de las capacidades organizacionales para enfrentar el riesgo.

Sin embargo, a pesar de las diferencias en el discurso y en el planteamiento inicial de los proyectos, las diferencias en el producto construido han sido poco tangibles. Más allá de algunas salvedades puntuales en el diseño, ambas gestiones metropolitanas han construido escaleras respondiendo al enfoque de priorizar la función de movilidad por encima de las diversas funciones que un espacio como la calle alberga. La habilitación parcial de la calle a través de la construcción de escaleras ha sido una característica común en ambas gestiones metropolitanas, y un proceder que resulta ser similar al de las iniciativas inconexas de las gestiones distritales y no muy distinta a las intervenciones aisladas por parte de la población.

Para sustentar las afirmaciones vertidas sobre lo formalmente similares que han sido las políticas urbanas respecto a la construcción de escaleras pese a los discursos aparentemente diversos, la investigación se propuso identificar un sector de estudio representativo que pudiese mostrar tangiblemente los hechos.

Por tanto, partiendo de la identificación de Ate como el distrito con mayor cantidad de proyectos de inversión pública orientados a la construcción de escaleras y analizando la data relacionada a sus zonas administrativas y sectores urbanos beneficiados a partir de filtros multinivel (Figura 6), se ha identificado que:

i. A nivel zonal, la zona administrativa del distrito de Ate que concentra la mayor cantidad de proyectos registrados es la Zona 06, con 62 de un total de 99 proyectos de escaleras.

ii. Considerando la necesidad de evaluar los proyectos que presenten algún nivel de certeza sobre su ejecución, se ha optado por evaluar solo los

8 Ítem solicitado para la inscripción y formulación del proyecto en el Banco del Proyectos del SNIP. 
proyectos que cuenten con expediente técnico. Con este criterio, la Zona 06 pasa de contar con 62 proyectos registrados a contar con 46 proyectos con expediente técnico.

iii. A nivel sectorial, los proyectos registrados y con expediente técnico en la Zona 06 se distribuyen casi equitativamente entre el sector de Huaycán (24 de 46) y el sector de Horacio Zeballos (22 de 46).

iv. Considerando la necesidad de evaluar los proyectos según su alcance, se ha optado por tener en cuenta como criterio final de selección la longitud total de las escaleras proyectadas. Con ese criterio, se ha determinado que Horacio Zeballos es el sector urbano de mayor representatividad, con un alcance de 4628,62 metros lineales de escaleras, frente a los 3950,11 de Huaycán.

Identificado Horacio Zeballos como el sector urbano más representativo, y a partir de trabajo de campo, se logró determinar que, hasta el ańo 2015, en el sector existían 110 escaleras que formaban parte de la retícula de calles del sector (Figura 7). De este total, 72 fueron construidas por el Estado mediante los 24 proyectos de inversión pública previamente identificados; y 38 fueron construidas por la población mediante autoconstrucción (Figura 8).

\subsection{Modalidades de habilitación}

Inversión pública y autoconstrucción son las modalidades de habilitación de escaleras presentes tanto en Horacio Zeballos como en casi todos los sectores urbanos donde se emplazan los «nuevos» barrios populares. Y si bien se puede percibir que la cantidad de intervenciones por inversión pública es notablemente mayor a las intervenciones mediante autoconstrucción, esto solo se debe a que el Estado, con el objeto de consolidar lo preexistente, ha intervenido sobre anteriores escaleras autoconstruidas por la población.

\section{Autoconstrucción}

Esta modalidad no asegura estándares en su producción. Las técnicas constructivas empleadas pueden resultar siendo muy artesanales (Figura 9, A, B, C) o encontrarse a nivel de la construcción formal (Figura 7, D). Los materiales empleados también pueden resultar muy diversos, como los tres tipos identificados en el sector: llantas rellenadas con tierra (Figura 9, A), bloques de piedra (Figura 9, B, C) y concreto encofrado (Figura 9, D). La elección tanto de las técnicas como de los materiales a ser empleados dependen directamente de los recursos disponibles para la construcción, los cuales siempre son variables. 


\section{Figura 6. Proyectos de inversión pública para la construcción de escaleras en las calles de Ate}

NIVEL ZONAL: ZONAS BENEFICIADAS
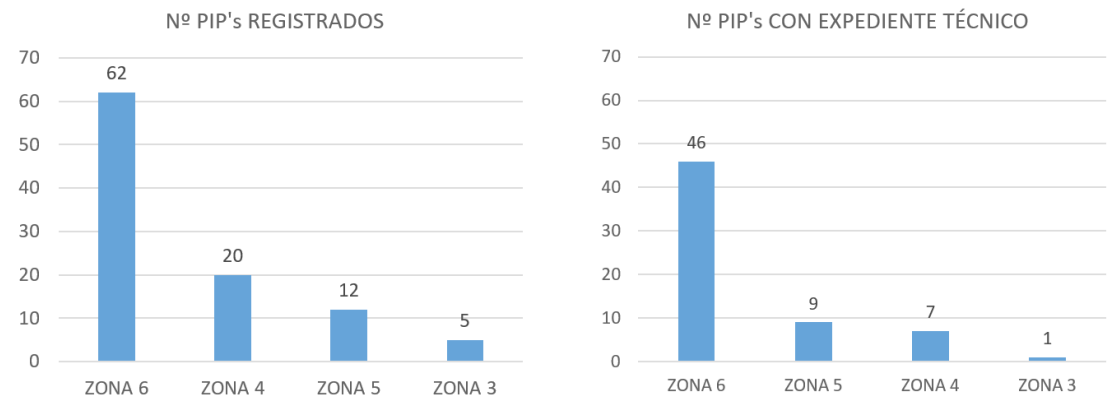

NIVEL SECTORIAL: SECTORES BENEFICIADOS

№ PIP's REGISTRADOS

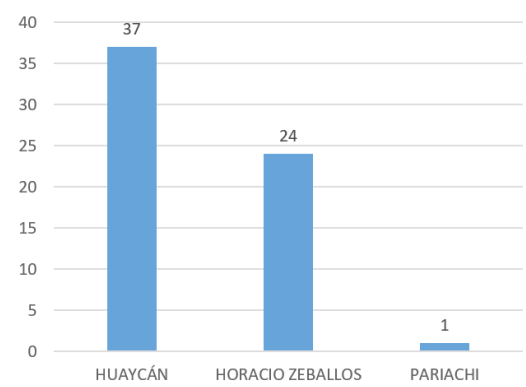

№ PIP's CON EXPEDIENTE TÉCNICO

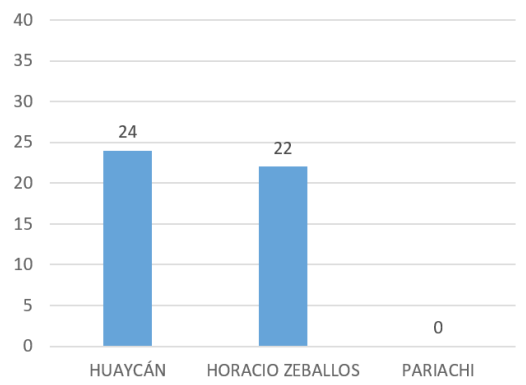

SEGÚN ALCANCE DEL PIP

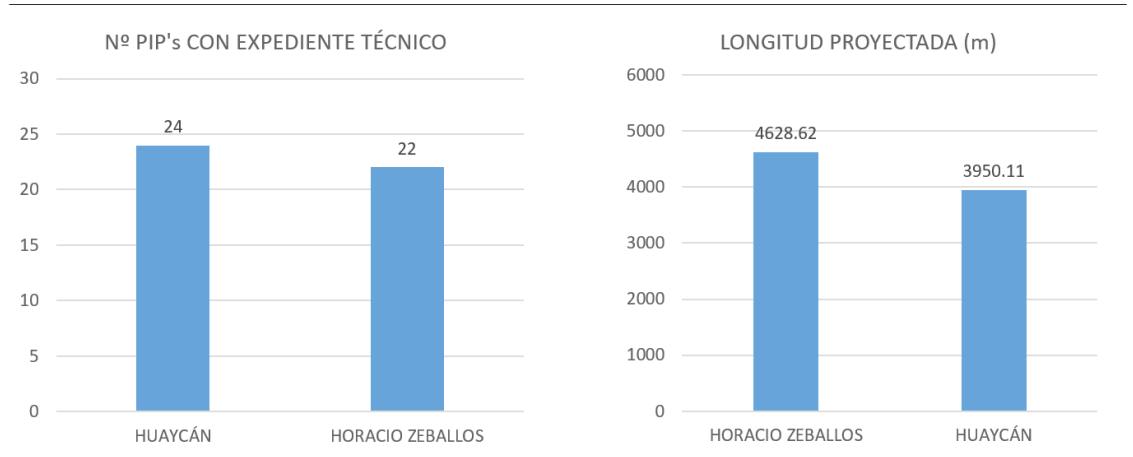

Fuente: Banco de Proyectos SNIP (fecha de corte a 2015). Elaboración propia. 
Figura 7. Mapeo de escaleras construidas en Horacio Zeballos

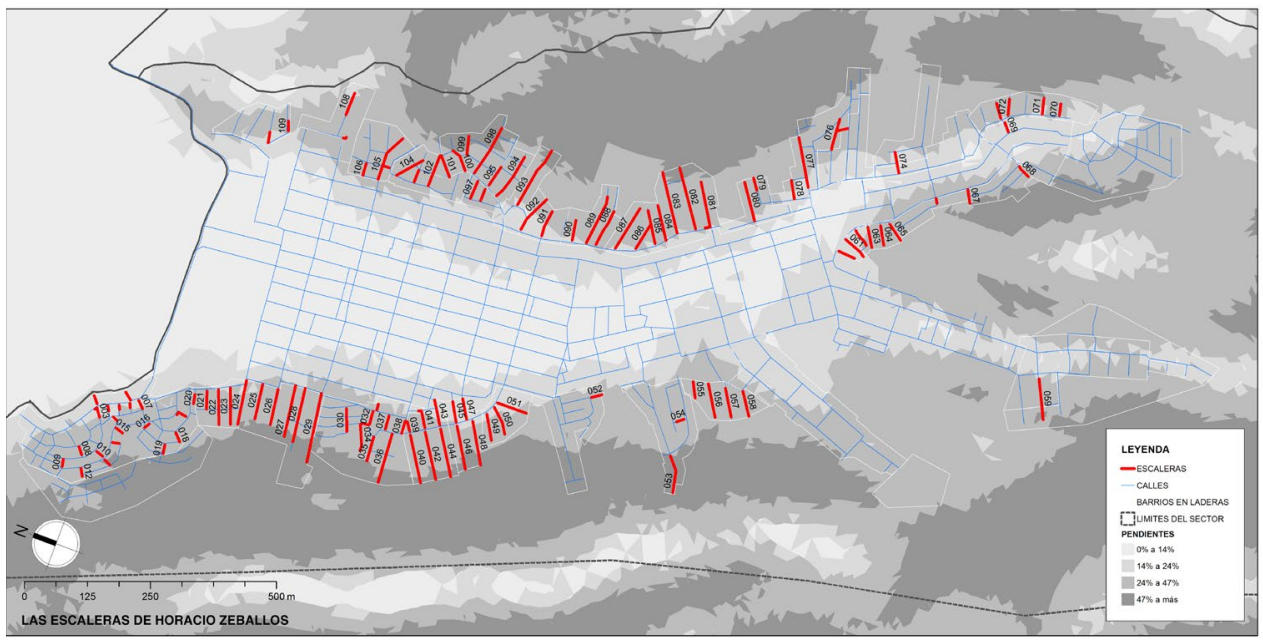

Fuente: Trabajo de campo (2015). Elaboración propia.

Figura 8. Mapeo de escaleras construidas en Horacio Zeballos, según modalidad de habilitación

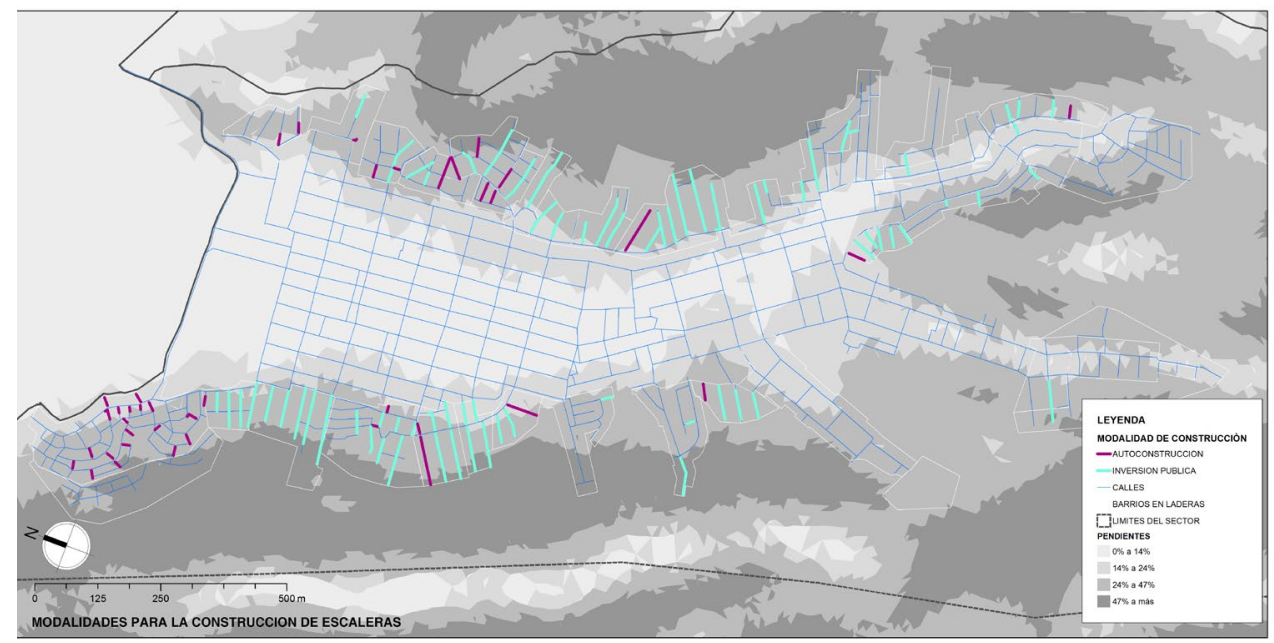

Fuente: Trabajo de campo (2015). Elaboración propia.

Bajo esta modalidad, en ocasiones se observan escaleras construidas cerca a otros elementos de habilitación urbana, como los muros de contención (Figura 9, C), ello puede tener dos razones: la escalera se implementa posterior al muro de contención, usándolo de elemento de apoyo que la vuelve más segura; o el 
muro de contención se implementa posterior a la existencia de la escalera, cumpliendo el objetivo de estabilizar el talud y brindar seguridad a los transeúntes.

Además, la presencia de mobiliario urbano es escasa o inexistente. En algunas ocasiones se ha identificado la presencia de barandas de madera - $\mathrm{O}$ estacas a manera de ellas - amarradas con soguillas y sin refuerzos diagonales adecuados. Sin embargo, su presencia no suele ser prioridad para los vecinos.

\section{Inversión pública}

Esta modalidad, en teoría, debería aportar con soluciones más adecuadas a fin de lograr mejoras significativas a las condiciones físicas previas. Sin embargo, las construcciones mediante esta modalidad se han caracterizado por el uso generalizado del concreto reforzado y vaciado in situ, materializándose a manera de escaleras sobrepuestas a la geografía, localizadas en el eje central de las calles y adquiriendo anchos que oscilan entre 1,20 m y 1,50 m de longitud. Esto ha significado una habilitación parcial de la calle.

El mobiliario urbano dotado por esta modalidad se puede clasificar en dos tipos: los funcionales y los ornamentales. Dentro de los funcionales, son omnipresentes las barandas de madera, cuya ubicación no siempre es la adecuada ni tiene en cuenta el ingreso a las viviendas, y cuyo mantenimiento es escaso o inexistente (Figura 9, E). Dentro de las ornamentales, se ha identificado la presencia de pérgolas al inicio o en la parte intermedia de las escaleras (Figura $9, F)$, que responde a la intención de una gestión municipal en particular por aportar, en alguna medida, a la mejora del espacio público (Susana Villarán, 2011-2014). Se las considera como elementos ornamentales porque no son constantes en todas las intervenciones de la gestión ni se encuentran en cada uno de los lugares de descanso a lo largo de las escaleras, como si debieran estar ubicadas si tuviesen un objetivo funcional.

Si bien la mayoría de las intervenciones del Estado ha optado por la habilitación de las calles que presenten mayor cantidad de beneficiarios para lograr un mayor impacto, también se han identificado algunos casos donde ese criterio no se ha aplicado con rigurosidad pues se ha intervenido en calles con escasa presencia de viviendas (Figura 9, G). Este tipo de intervenciones permite cuestionamientos sobre las reales intenciones de algunas gestiones municipales respecto a la construcción de escaleras, intenciones que no son consistentes con la búsqueda del beneficio común y la adecuada administración de recursos públicos.

Por último, las intervenciones del Estado — si bien se ven bastante limitadas y fiscalizadas por el sistema de inversión pública (antes denominado SNIP y ahora Invierte) — deberían de caracterizarse por la calidad técnica de 
su propuesta y ejecución, por contar con profesionales capacitados y por hacer buen uso del dinero público. Más allá de la cuestión misma de habilitar parcialmente las calles con escaleras, no siempre la ejecución de la obra es la más adecuada. Incluso se han identificado varios casos donde la solución brindada por el Estado es deficiente, insegura y de pésima calidad (Figura 9, H).

Figura 9. Horacio Zeballos: escaleras según modalidades de habilitación

AUTOCONSTRUCCIÓN

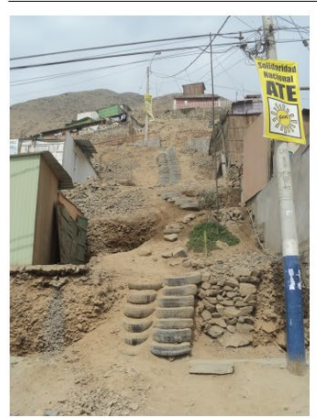

[A]

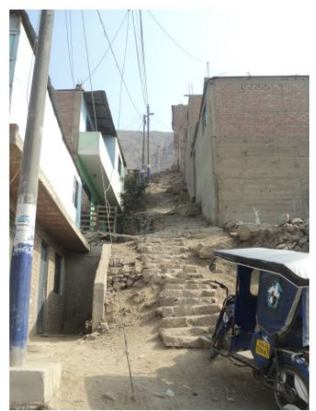

[B]

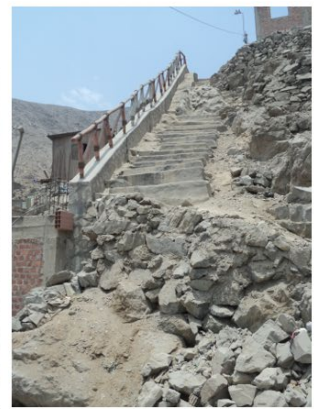

[C]

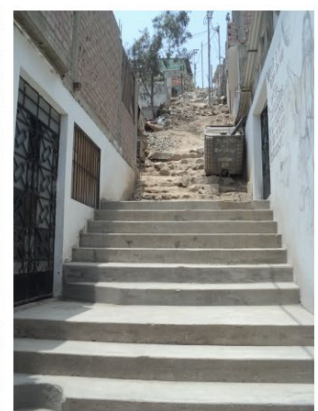

[D]

INVERSIÓN PÚBLICA

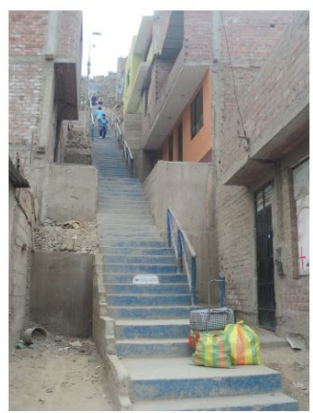

[E]

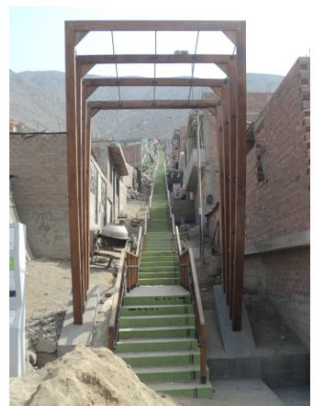

[F]

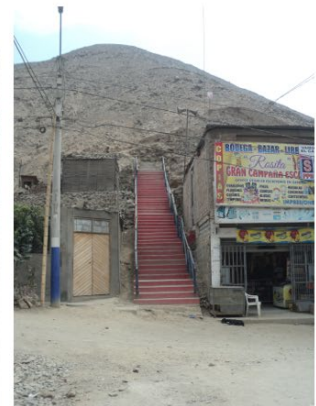

[G]

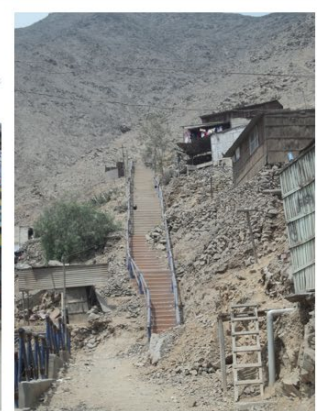

[H]

Fuente: Archivo propio, 2015. Elaboración propia.

\subsection{Entes gestores de la habilitación}

Las modalidades de habilitación están relacionadas a los entes que la gestan. Por un lado, la autoconstrucción implica necesariamente que la gestión para tal fin estuvo a cargo de la población, desde la concepción de la necesidad, el recaudo de recursos y la ejecución misma, independientemente si ha sido elaborado por trabajo comunitario o por contrato externo. Por otro lado, la inversión pública puede darse desde todos los sectores del Estado. Para el caso de la construcción de escaleras en los «nuevos» barrios populares, al ser habilitaciones de vías 
locales, las competencias recaen directamente en los Gobiernos locales; y al ser un caso que se evidencia a lo largo de la periferia de la ciudad, también ha estado considerado dentro de las competencias del Gobierno regional.

Tres son los entes gestores que han intervenido en Horacio Zeballos mediante la construcción de escaleras: la población, la Municipalidad Distrital de Ate y la Municipalidad Metropolitana de Lima. Sin embargo, se han encontrado casos donde los esfuerzos del municipio distrital han coincidido con los del municipio metropolitano, ocasionando que ciertas construcciones sean producto de ambas gestiones — a manera de híbridos-, hecho que revela errores de comunicación entre los municipios y errores en el sistema de inversión pública.

Las gestiones municipales, como entes gestores de habilitación de escaleras, han evidenciado una particular preocupación por dejar su «huella institucional» en las intervenciones realizadas, ya sea mediante el color usado para pintar las escaleras o a través de señalética de contundente visibilidad. Sin embargo, se cuestiona si estas «huellas» han sido trascedentes o apenas una alegoría proselitista.

Tres son las gestiones involucradas en la construcción de escaleras en Horacio Zeballos: las gestiones de los alcaldes Juan Dupuy García y Óscar Benavides Majino a nivel de municipio distrital, y la gestión de Susana Villarán a nivel de municipio metropolitano.

La gestión distrital del alcalde Dupuy se ha caracterizado por pintar las escaleras y las barandas de color celeste y colocar monolitos al costado de la escalera o cerámicos en los peldaños con inscripciones acerca del municipio, el proyecto de inversión pública y el nombre del alcalde (Figura 10, A). Con una estrategia parecida, la gestión distrital del alcalde Benavides se ha caracterizado por pintar las escaleras de color rojo y las barandas de color azul y colocar monolitos al costado de la escalera con inscripciones con información relacionada a su gestión (Figura 10, B).

La gestión metropolitana de la alcaldesa Susana Villarán no ha sido la excepción a la regla, pintando las escaleras de color verde y colocando monolitos al costado de las escaleras. Cabe mencionar que, en este caso, las inscripciones en los monolitos no solo incluían información sobre el municipio sino también acerca del asentamiento humano y la calle en cuestión, intentando servir como elemento para facilitar la ubicación. Además, se tenía la intención de llegar a un sector vulnerable de la población a través de presentar esta información también en sistema Braille. Sin embargo, más allá de las buenas intenciones, los monolitos siguieron siendo percibidos por la población como elementos proselitistas debido a sus características estéticas de directa alegoría a la gestión Villarán (Figura 10, C).

Por lo descrito se hace evidente que las diferencias físicas entre las intervenciones de una y otra gestión radican básicamente en características externas a 
la construcción de la escalera per sé. En algunos casos, las diferencias son estéticas, como las variaciones en el color o el diseño de los elementos alegóricos a la gestión de turno. En otros casos, las diferencias responden a prioridades transversales, como la inclusión de inscripciones en sistema Braille o la instalación esporádica de pérgolas. Sin embargo, estas diferencias no repercuten ni directa ni significativamente en la mejora de la calidad física de la calle como consecuencia de su habilitación.

Finalmente, con lo que respecta a las escaleras híbridas, sus características no han sido particulares ni su procedencia anónima. Por el contrario, a raíz de las «huellas institucionales» dejadas por cada gestión municipal — como el pintar un tramo de color rojo y el otro de verde-, se pone en evidencia que estas escaleras han sido resultado de la intervención de las gestiones del alcalde distrital Benavidez y la alcaldesa metropolitana Villarán (Figura 10, D).

La presencia de escaleras híbridas, como las mostradas, evidencian dos cosas: la poca coordinación interinstitucional entre el Gobierno metropolitano y el Gobierno distrital, lo que deriva en duplicidad de esfuerzos y recursos; y, el poco control que el SNIP tenía respecto a la verificación de la ubicación exacta de los proyectos elaborados bajo su metodología.

Figura 10. Horacio Zeballos: escaleras según entes gestores de la habilitación

ESTADO COMO ENTE GESTOR
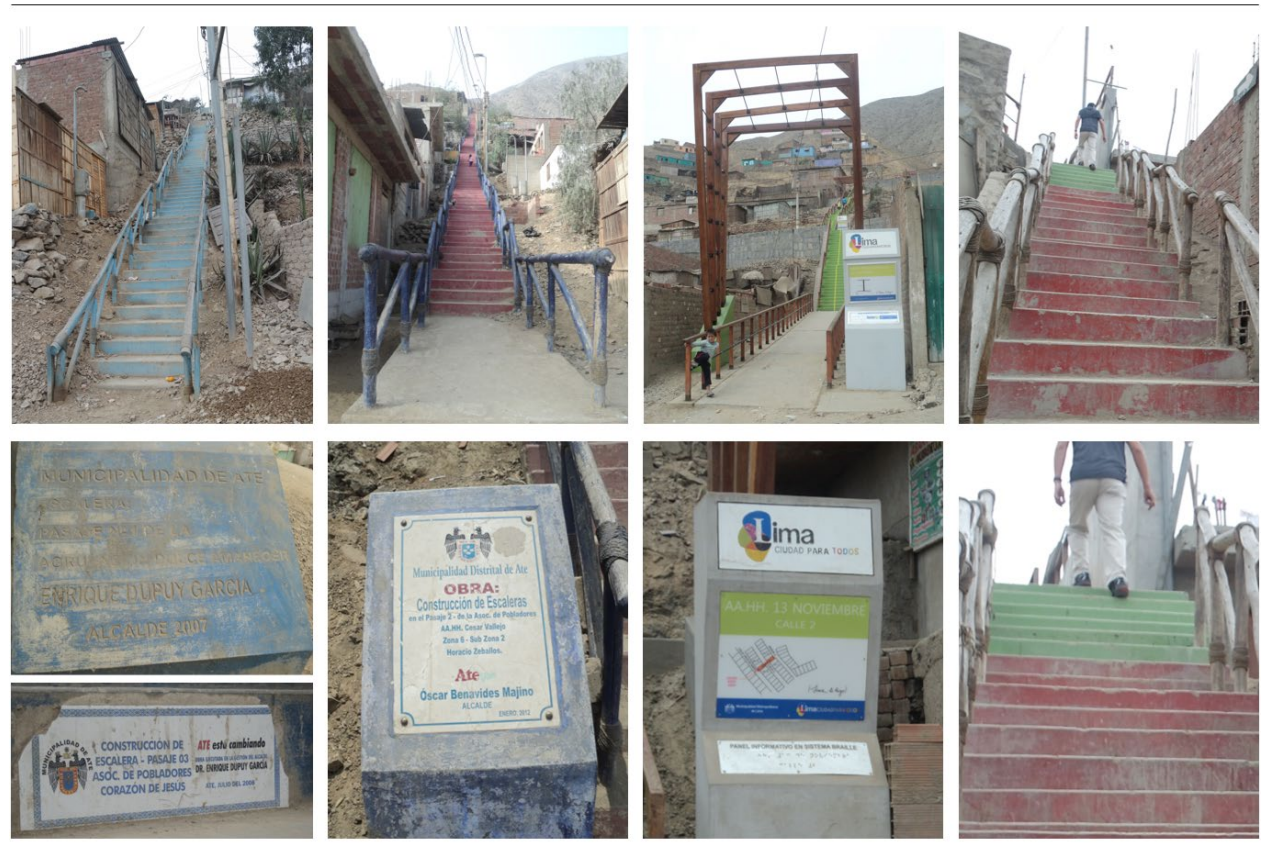

[A]

[B]

[C]

[D]

Fuente: Archivo propio, 2015. Elaboración propia. 


\section{LA ESCALERA ES LA CALLE}

Más allá de las implicancias que presenta la construcción de escaleras en sus diversas modalidades, la vida barrial que se desarrolla en torno a ellas no se ve condicionada solo a sus características físicas. El tener en cuenta que el uso y la apropiación del espacio barrial, tal como afirma Ladizesky (2011), es mayor cuando los ingresos de los habitantes de un barrio son menores, conlleva a entender por qué en los nuevos barrios populares la calle se habita independientemente de si se habilita o no. Tanto el espacio de las escaleras como los espacios intersticiales entre ellas y las viviendas aledañas son escenario de actividades que dinamizan la vida barrial, trascendiendo lo infraestructural y dotando a cada calle de una identidad particular.

Por ejemplo, algunos vecinos aprovechan los intersticios entre escalera y vivienda para acondicionar espacios a manera de terrazas o jardines, optando por el uso público y recreativo del espacio - aunque este a veces se encuentre cercado (Figura 11, A)—. En otros casos, estos espacios son acondicionados como extensión del uso comercial y productivo a pequeña escala de viviendas y tiendas locales, optando así por el uso productivo del espacio (Figura 11, B).

La escalera también es usada como espacio de juego. Sus características físicas la vuelven atractivas para los niños, quienes ingeniosamente encuentran en barandas y peldańos una multiplicidad de usos (Figura 11, C). Además, las actividades de socialización siempre están presentes. A pesar de la habilitación incompleta de la calle, los vecinos encuentran la manera de apropiarse de sus particularidades, como sus espacios residuales y elementos inacabados, llegando incluso a interactuar con ellas de formas poco ortodoxas, como escalándolas o atravesándolas de forma vertical a falta de una conexión directa (Figura 11, D).

Sin embargo, si bien el dinamismo barrial observado en estos sectores urbanos depende más de sus factores socioeconómicos que de sus condiciones físicas, no significa que estas últimas no sean importantes. Como sostiene Gehl (2003), mientras mejores sean las condiciones físicas del espacio, más diversas y seguras serán las actividades desarrolladas en él. Por eso la importancia de revisar la forma actual de producción de escaleras, desde su formulación hasta su construcción, para así poder rediseñarlas en función a no solo lo que el marco normativo le permite hacer al Estado, sino también a lo que la realidad de estos «nuevos» barrios y de sus habitantes requiere realmente. 
Figura 11. Usos y actividades en torno a las escaleras en los «nuevos» barrios

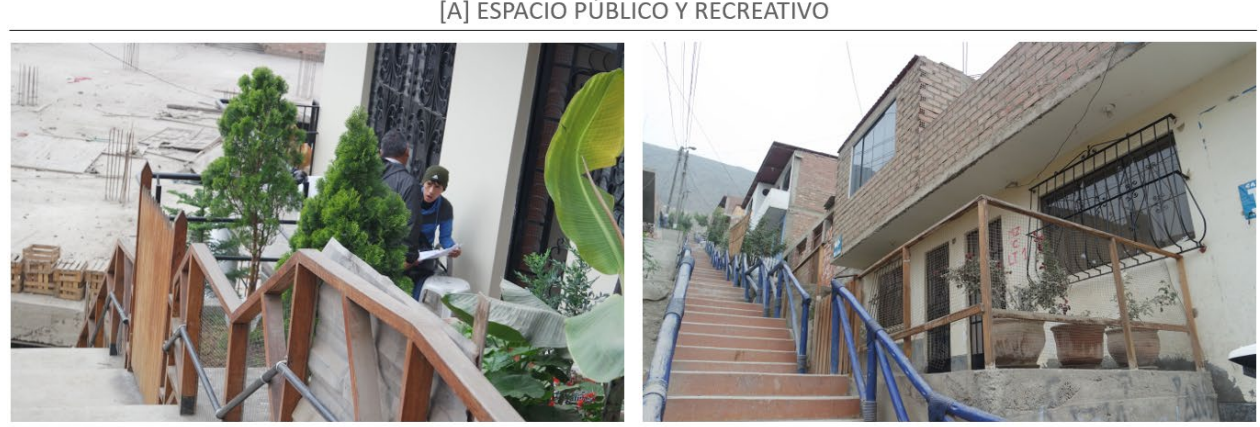

[B] ESPACIO PRODUCTIVO / COMERCIAL
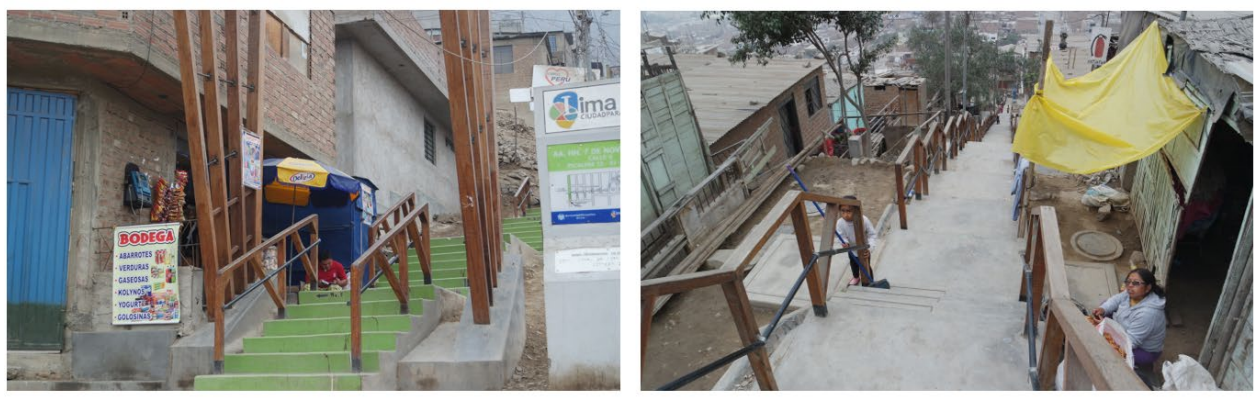

[C] ESPACIO DE SOCIALIZACIÓN Y DE USOS PARTICULARES
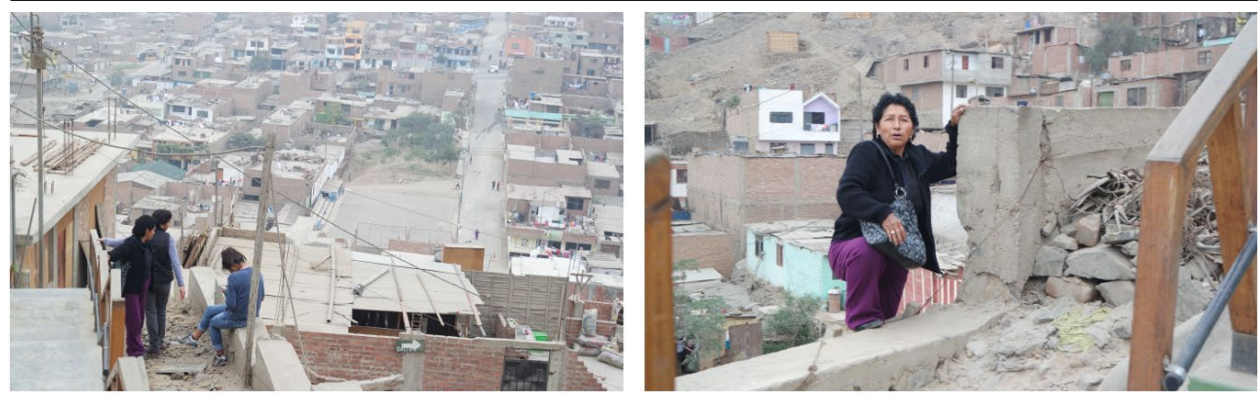

[D] ESPACIO LÚDICO
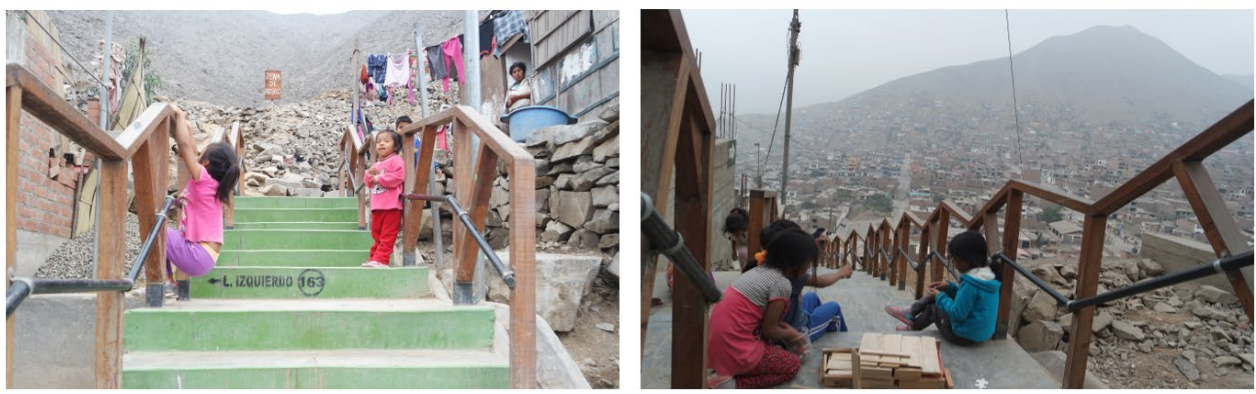

Fuente: Archivo propio, 2015. Elaboración propia. 


\section{Conclusiones}

Las escaleras en los nuevos barrios populares comenzaron a ser construidas debido a la necesidad de la población de acceder a las viviendas emplazadas en geografías complicadas, característica inherente a la conformación de estos barrios. Al inicio fueron implementadas por la población a través de la autoconstrucción y posteriormente el Estado adoptó esta práctica y la institucionalizó como política urbana para intervenir en laderas a través de proyectos de inversión pública.

La inversión del Estado destinada a la construcción de escaleras, tanto a nivel local como regional, en general se ha caracterizado por ser de poco alcance — con una mayor cantidad de proyectos formulados a partir de proyectos de inversión pública menores con montos de inversión inferiores a los S/ 300 000,00. Sin embargo, la evidencia muestre que el gobierno regional de Lima Metropolitana, en comparación con los gobiernos locales, tiene en su haber una cantidad significativa de proyectos de mayor alcance y complejidad — proyectos de inversión pública menores que superan los $S / 300$ 000,00 y proyectan mayor número de escaleras- que sumados representan la mitad del total de sus proyectos formulados. Esta característica, aunque compatible con la mayor capacidad de gestión y de impacto que presenta el gobierno regional, lamentablemente no ha llegado a ser el formato de inversión más representativo, al menos no hasta el cierre de la presente investigación.

La construcción de escaleras se ha encontrado supeditada a los discursos y las estéticas correspondientes a las gestiones municipales de turno. Como resultado, las diferencias sustanciales en el producto construido han sido poco tangibles. Más allá de algunas salvedades puntuales en el diseño ornamental, las escaleras han sido construidas respondiendo al mismo enfoque de priorizar la función de movilidad por encima de las diversas funciones que un espacio como la calle alberga. La construcción de escaleras en los nuevos barrios populares ha concluido en una habilitación parcial de las calles, resultado impensado como solución en cualquier otra parte de la ciudad.

La vida barrial que se desarrolla en torno a las escaleras en los nuevos barrios populares depende más de los factores socioeconómicos de su población —que la impulsa a utilizar la calle de manera más intensiva- que de sus condiciones físicas. Pero esta situación no acontece por elección sino como resultado de la carencia. El entorno construido en ningún momento deja de ser importante, por el contrario, es aún más apremiante para el Estado proporcionar respuestas eficientes que apunten a la mejora de esta situación, para cumplir con su objetivo de velar por el bienestar de sus vecinos y de sus barrios. 


\section{REFERENCIAS}

Barreda, J. (2012). Identificación de zonas de riesgo por pendiente en las zonas periféricas de lima metropolitana [Consultoría no publicada para la Municipalidad Metropolitana de Lima].

Barreda, J. (2014a, marzo). Hacia un sistema de información de la ciudad. Arkinka, 18(220), 54-57.

Barreda, J. (2014b, setiembre). Área Pendiente Urbe [Base de datos no publicada].

Barreda, J. y Ramírez Corzo, D. (2004). Lima: Consolidación y expansión de una ciudad popular. En Perú hoy: las ciudades en el Perú (pp. 199-218). Lima: Desco.

Gehl, J. (2003). La humanización del espacio urbano, la vida social entre los edificios (5a ed.). Barcelona, España: Editorial Reverté.

Ladizesky, J. (2011). El espacio barrial. Buenos Aires: Consejo Profesional de Arquitectura y Urbanismo. 